doi: $10.15407 /$ ujpe61.06.0537

A.D. SUPRUN, L.V. SHMELEVA

Taras Shevchenko National University of Kyiv

(64/13, Volodymyrs'ka Str., Kyiv 01601, Ukraine,; e-mail: lshmel@univ.kiev.ua)

\title{
SOME ASPECTS OF GENERALIZED DYNAMICS OF QUASIPARTICLES IN CRYSTALS WITH UNIT CELL OF ARBITRARY COMPLEXITY
}

\begin{abstract}
The conditions, under which the general description of the dynamical properties of quasiparticles is almost identical with those of real relativistic particles, are analyzed. Such analysis is, especially, actual today in connection with the growing interest in electronic properties of graphene and other nanostructures of carbon origin (fullerenes, nanotubes, etc.). The development of the traditional applications of quasiparticles (superfluidity, transfer of charge or energy) also requires a generalized analysis of dynamical properties of quasiparticles. The problem of the correlation of quantum and classical methods of description of the quasiparticles in the case of the excited states of crystals is considered. In order to focus attention on the discussed problem, the obtained results are demonstrated on the example of electronic excitations of crystals in the simplest case where other effects are neglected (phonons, defects, high density of excitations, which would require the account for interactions between them, the response of a lattice to excitations, and so forth). It is shown that such excitations can be described in three ways simultaneously. The first is the quantum description of the examined excitations in terms of wave functions and eigenvalues of energy. The second method is classical. It arises from the quantum method and is formulated in terms of the wave momentum. The third method, which follows from the second one, is also a description of the classical type, but is related to the other momentum - the mechanical one. The latter descriptions (the third or second one) make it possible to interpret the experimental data in terms of the usual relativistic dynamics.

Keywords: quasiparticles, dispersive dependence, relativistic approximation, dynamical Dirac model, graphenes.
\end{abstract}

\section{Introduction}

Electronic properties of graphene and other nanostructures of the carbon series require a general analysis of dynamical properties of quasiparticles. Their field of applications is from superconductivity [1] and superfluidity [2] up to solitary excitation transfer processes [3-9]. In particular, the processes of such transfer are normally associated in practice with the spatial transmission of charge or energy. But they can also be used as information signals. Studying the fundamental properties of quasiparticles (especially dynamical properties) is important as for traditional processes [3-6], so for such updated problem as the properties of graphene [10] in, particularly, the Dirac dynamical model [11]. This is important also for a more complete understanding of the physical proper-

(c) A.D. SUPRUN, L.V. SHMELEVA, 2016

ISSN 2071-0194. Ukr. J. Phys. 2016. Vol. 61, No. 6 ties of real particles, especially, in connection with the mentioned dynamical Dirac model in graphenes [12].

The analysis of the general dynamical properties of free quasiparticles is fulfilled on the basis of one of the major characteristics of the excited states of condensed matter. This is the dispersive dependence of the energy or frequency on a wave vector [1-15]. We will also examine the general conditions, under which the dynamical Dirac model becomes real. The problem of relationship between quantum and classical methods of description of quasiparticles is considered as well.

\section{Materials and Methods. Common Remarks About Dynamical Properties of Quasiparticles in Crystals}

\subsection{Basic relations}

We will analyze the dependence $E(\mathbf{k}) \equiv E\left(k_{1}, k_{2}, k_{3}\right)$. Since the wave vector $\mathbf{k}$ is always associated with the 
wave momentum $\mathbf{P}=\hbar \mathbf{k}$, the quantity $E(\mathbf{k})$ acquires at once the sense of Hamiltonian: $E(\mathbf{k}) \equiv H(\mathbf{P})$. In this case, it is possible to find a speed of some classic object, $V_{i}(\mathbf{k})=\frac{\partial H(\mathbf{P})}{\partial P_{i}} \equiv \frac{1}{\hbar} \frac{\partial E(\mathbf{k})}{\partial k_{i}} \equiv \frac{\partial \omega(\mathbf{k})}{\partial k_{i}}$, and its $\operatorname{mass} m_{i j}^{-1}(\mathbf{k})=\frac{\partial V_{i}}{\partial P_{j}} \equiv \frac{1}{\hbar} \frac{\partial V_{i}(\mathbf{k})}{\partial k_{i}} \equiv \frac{1}{\hbar^{2}} \frac{\partial^{2} E(\mathbf{k})}{\partial k_{i} \partial k_{j}} \equiv \frac{\partial V_{j}}{\partial P_{i}}$. The expressions $E(\mathbf{k})$ and $\mathbf{V}(\mathbf{k})$ are parametric dependences of the energy on the speed. If formulate the energy as a function of the speed, it immediately gets a status of the mechanical Lagrangian: $E(\mathbf{k}) \equiv$ $\equiv L_{m}(\mathbf{V})$, which determines the components of the mechanical momentum $\mathbf{P}_{m}: P_{i(m)}=\frac{\partial L_{m}(\mathbf{V})}{\partial V_{i}}$. Here, the index determines the Cartesian components of the corresponding values. In [16], it was shown that this is indeed a mechanical momentum, inasmuch as determined by the equality: $P_{i(m)}(\mathbf{k})=m_{i j}(\mathbf{k}) V_{j}(\mathbf{k})$. As is obvious from the last equation, the momentum $\mathbf{P}_{m}$ differs from a momentum $\mathbf{P}$, at least, by two circumstances: by the determination and the different relation to the mass.

The presence of two specifications of the classical type for a free quasiparticle, which are based on the equalities $E(\mathbf{k}) \equiv H(\mathbf{P}) \equiv L_{m}(\mathbf{V})$, generates an additional duality of the classic-classic type. Between these two descriptions, there exists another "intersection", which supplements this equality: both descriptions have identical speed.

Here, we want to focus our attention on that the dynamical Dirac model is universal for any points of the $\mathbf{k}$-space (except for the points of extrema) and for any crystals, but not only for graphenes in vicinities of the points $k_{i} \sim \pi / 2$. For clarity of the presentation of this problem, we have to give briefly some of the main aspects of the dynamical description of quasiparticles, which were discussed in [16] in detail.

\subsection{Basic relations in crystals}

\subsubsection{Simple unit cell}

Without regard for a reaction of the lattice to the excitation [17], the typical Hamiltonian for singleelectronic excitations in simplest solids is determined by the equality $[3-6,16,18]$

$$
\begin{aligned}
& E(\{a\})=\frac{1}{2}\left\{\sum_{\mathbf{n} \mathbf{l}}^{\prime} w_{\mathbf{n}, \mathbf{n}+\mathbf{l}}+\sum_{\mathbf{n}} 2 D_{\mathbf{n}}\left|a_{\mathbf{n}}\right|^{2}+\right. \\
& \left.+\sum_{\mathbf{n} \mathbf{l}}^{\prime} M_{\mathbf{n}, \mathbf{n}+\mathbf{l}}\left(a_{\mathbf{n}}^{*} a_{\mathbf{n}+\mathbf{l}}+a_{\mathbf{n}+\mathbf{l}}^{*} a_{\mathbf{n}}\right)\right\} .
\end{aligned}
$$

The vectors $\mathbf{n}$ and $\mathbf{l}$ determine the spatial position of a separate atom or molecule. The factor $a_{\mathbf{n}}$ is an unknown part of the wave function of the electronic subsystem of a crystal and is determined by the condition of dynamical minimization of functional (1) $[4,16]$. The eigenvalue $E(\mathbf{k})$ is determined simultaneously with $a_{\mathbf{n}}$. The matrix element $w_{\mathbf{n}, \mathbf{n}+\mathbf{l}}$ determines the interaction between atoms or molecules. The matrix element $D_{\mathbf{n}}$ is the energy, which determines the interaction between the crystal and an external excitation. The matrix element $M_{\mathbf{n}, \mathbf{n}+\mathbf{l}}$ determines the energy of the resonant exchange interaction. These elements are determined in details in $[16,17]$. The condition of dynamical minimization of functional (1) is equivalent to the procedure of reduction to the diagonal type of the operator proper to (1) [5]. This procedure enables one to find the energy $E(\mathbf{k})$ directly at the operator level.

In the simplest case, the potential energy $D_{\mathbf{n}}$ does not depend on the variable $\mathbf{n}: D_{\mathbf{n}}=D_{\mathbf{0}}$, and functional (1) takes a simpler form:

$E(\{a\})=E_{0}+\frac{1}{2} \sum_{\mathbf{n} \mathbf{l}}^{\prime} M_{\mathbf{n}, \mathbf{n}+\mathbf{l}}\left(a_{\mathbf{n}}^{*} a_{\mathbf{n}+\mathbf{1}}+a_{\mathbf{n}+\mathbf{l}}^{*} a_{\mathbf{n}}\right)$.

Here, $E_{0} \equiv U_{0}+D_{\mathbf{0}} N$, where $U_{0} \equiv(1 / 2) \sum_{\mathbf{n} \mathbf{l}}^{\prime} w_{\mathbf{n} \mathbf{l}}$. The common determination of norm is used: $N \equiv$ $\equiv \sum_{\mathbf{n}}\left|a_{\mathbf{n}}\right|^{2}$. Using the procedure of Hamilton dynamical minimization, $i \hbar \frac{\partial a_{\mathbf{n}}}{\partial t}=\frac{\partial E(\{a\})}{\partial a_{\mathbf{n}}^{*}}$, it is possible to get the equation

$i \hbar \frac{\partial a_{\mathbf{n}}}{\partial t}=-\frac{1}{2} \sum_{\mathbf{l}}^{\prime}\left|M_{\mathbf{l}}\right|\left(a_{\mathbf{n}+\mathbf{l}}+a_{\mathbf{n}-\mathbf{l}}\right)$.

Here, two circumstances were taken into account [16]. First, the matrix elements $M_{\mathbf{n}, \mathbf{n}+\mathbf{1}}$ under the conditions of ideal crystal depend only on the difference of lower indices. Second, in typical crystals, they are negative. Then functional (2) becomes

$E(\{a\})=E_{0}-\frac{1}{2} \sum_{\mathbf{n} \mathbf{l}}^{\prime}\left|M_{\mathbf{l}}\right|\left(a_{\mathbf{n}}^{*} a_{\mathbf{n}+\mathbf{l}}+a_{\mathbf{n}+\mathbf{1}}^{*} a_{\mathbf{n}}\right)$.

Applying the formal operator identity $a_{\mathbf{n} \pm \mathbf{l}} \equiv$ $\equiv \exp \left\{ \pm\left(\mathbf{l} \cdot \nabla_{\mathbf{n}}\right)\right\} a_{\mathbf{n}}$ to Eq. (3), we see that it takes form of the Schrödinger equation: $i \hbar \frac{\partial a_{\mathbf{n}}}{\partial t}=\widehat{H} a_{\mathbf{n}}$, where

$\widehat{H} \equiv-\sum_{\mathbf{l}}^{\prime}\left|M_{\mathbf{l}}\right| \operatorname{ch}\left(\mathbf{l} \cdot \nabla_{\mathbf{n}}\right)$.

As system (3) is complex, the function $a_{\mathbf{n}}$ must be represented firstly in a most general form [19]:

$a_{\mathbf{n}}(t)=\varphi_{\mathbf{n}}(t) \exp \left[i \Gamma_{\mathbf{n}}(t)\right]$.

ISSN 2071-0194. Ukr. J. Phys. 2016. Vol. 61, No. 6 
Let us substitute (6) into (3) and separate the real and imaginary parts from each other. Then system (3) disintegrates into two subsystems for the functions $\varphi_{\mathbf{n}}$ and $\Gamma_{\mathbf{n}}$.

The first approximation, which results in the dynamics of quasiparticles identical to the dynamics of a free relativistic particle, is the approximation of a plane wave in a phase: $\Gamma_{\mathbf{n}}=\mathbf{k} \cdot \mathbf{n}-\omega t$, and a condition of unallocated amplitude: $\varphi_{\mathbf{n}}(t)=1 / \sqrt{N_{\mathrm{a}}}$, where $N_{\mathrm{a}}$ is the number of atoms in the excited region of a crystal. In this case, (6) takes the form

$a_{\mathbf{n}}(t)=\frac{1}{\sqrt{N_{\mathrm{a}}}} \exp [i(\mathbf{k} \cdot \mathbf{n}-\omega t)]$.

The substitution of (7) in energy (4) gives a law of dispersion for the examined case (absence of a reaction of the lattice on an excitation [17]):

$\varepsilon(\mathbf{k})=-\sum_{\mathbf{l}}^{\prime}\left|M_{\mathbf{l}}\right| \cos (\mathbf{k} \cdot \mathbf{l}) \equiv \hbar \omega(\mathbf{k})$

where $\varepsilon(\mathbf{k}) \equiv E(\mathbf{k})-E_{0}$. The comparison of expression (8) with the definition of operator (5) shows that the wave vector $\mathbf{k}$ can be put into accordance with the operator $-i \nabla_{\mathbf{n}}$ only ("minus" corresponds to the physical correctness of this accordance). As the wave vector $\mathbf{k}$ straightly determines a wave momentum $\mathbf{P}$ only, it is possible to conclude that, in the transition from the classical description to the quantum one, the operator of gradient is equivalent only to the wave momentum in the sense of the equality $\widehat{\mathbf{P}}=-i \hbar \nabla_{\mathbf{n}}$, but not to a mechanical momentum, which is determined by the equality $P_{i(m)}(\mathbf{k})=m_{i j}(\mathbf{k}) V_{j}(\mathbf{k})$ and is given in Subsection 2.1.

Next, the second and third approximations, which result into the dynamics of quasiparticles identical to the dynamics of a free relativistic particle, are the approximation of the nearest neighbors typical of crystals and the approximation of cubic lattice. In these approximations, the lattice vector takes the form $\mathbf{b}_{i} \equiv b \mathbf{e}_{i}\left(\mathbf{e}_{i}\right.$ are unit vectors of the Cartesian system), and energy (8) is simplified to the equality

$\varepsilon(\mathbf{k})=-|M| \sum_{i} \cos \left(b k_{i}\right)$.

A new notation is introduced: $M_{b \mathbf{e}_{i}} \equiv M$. Using the definitions of speed and mass, which are given in Subsection 2.1, we have

$V_{i}=\frac{1}{\hbar} \frac{\partial \varepsilon(\mathbf{k})}{\partial k_{i}}=C \sin \left(b k_{i}\right) ;$ $m_{i j}=\delta_{i j} \frac{m}{\cos \left(b k_{i}\right)} \equiv \delta_{i j} \frac{m}{\sqrt{1-\beta_{i}^{2}}}$.

Here, the standard notation of the relativistic dynamics is used: $\beta_{i} \equiv V_{i} / C$ are the components of a vector $\boldsymbol{\beta}$. For the dimensionless speed, formula (10) yields

$\beta_{i}=\sin \left(b k_{i}\right)$.

In (10), a constant $C$ has the dimension of speed and is determined by the equality

$C \equiv \frac{b|M|}{\hbar}$

It has a sense of the maximum possible speed of a quasiparticle under study. In (11), a constant $m$ has the dimension of mass and is defined by the equality

$m \equiv \frac{\hbar^{2}}{|M| b^{2}}$

It has the sense of a nondynamical part of the mass, i.e., it is independent of the wave vector $\mathbf{k}$ ). For the mass $m$, we formulate another useful definition:

$m \equiv \frac{\hbar}{b C}$

The result given in (11) is new for the quasiparticles.

In the approximations of nearest neighbors and, especially, cubic lattice, the tensor of mass becomes diagonal. Moreover, due to the approximation of cubic lattice, the tensor nature of mass is determined only by its dynamical properties. A constant $m$ is a scalar in these approximations. From the viewpoint of the relativistic approximation in the dynamics of quasiparticles, the equality $m C^{2}=|M|$, which can be got from (13) and (14), is of importance. In this case, energy (9) can be represented as

$\varepsilon(\mathbf{k})=-m C^{2} \sum_{i} \cos \left(b k_{i}\right)$,

and the identity $E(\mathbf{k}) \equiv H(\mathbf{P})$ follows from the equality

$\varepsilon(\mathbf{k}) \equiv H(\mathbf{P})=-m C^{2} \sum_{i} \cos \left(\frac{b}{\hbar} P_{i}\right)$. 


\subsubsection{General case}

In the general case of a complex unit cell (with several atoms), functional (1) has the form [20]:

$$
\begin{aligned}
& E(\{a\})=\frac{1}{2}\left\{\sum_{\mathbf{n}_{\alpha} \mathbf{m}_{\beta}}^{\prime} w_{\mathbf{n}_{\alpha} \mathbf{m}_{\beta}}+\sum_{\mathbf{n}_{\alpha}} 2 D_{\mathbf{n}_{\alpha}}\left|a_{\mathbf{n}_{\alpha}}\right|^{2}+\right. \\
& \left.+\sum_{\mathbf{n}_{\alpha} \mathbf{m}_{\beta}}^{\prime} M_{\mathbf{n}_{\alpha} \mathbf{m}_{\beta}}\left(a_{\mathbf{n}_{\alpha}}^{*} a_{\mathbf{m}_{\beta}}+a_{\mathbf{m}_{\beta}}^{*} a_{\mathbf{n}_{\alpha}}\right)\right\} .
\end{aligned}
$$

Here, the vectors $\mathbf{n}_{\alpha}, \mathbf{m}_{\beta}$ are determined by the relations:

$\mathbf{n}_{\alpha}=\mathbf{n}+\mathbf{r}_{\alpha}$,

where $\mathbf{n}=n_{i} \mathbf{b}_{i}$ is an ordinary vector of the lattice. Index $i$ runs from one up to three, by depending on the dimension of the crystal, and $n_{i}$ are integers corresponding to the number of unit cells: $n_{i}=0, \pm 1, \pm 2, \ldots$. The lattice vectors $\mathbf{b}_{i}$ are given by $\mathbf{b}_{i}=b_{i \xi} \mathbf{e}_{\xi}$, where $b_{i \xi}$ are projections of the vectors $\mathbf{b}_{i}$ on the Cartesian unit vectors $\mathbf{e}_{\xi}=\left(\mathbf{e}_{x}, \mathbf{e}_{y}, \mathbf{e}_{z}\right)$. Indices $\alpha$ and $\beta$ take integer values from 0 to $S-1$, where $S$ is the number of atoms in the unit cell. The zero values (e.g., $\alpha=0$ ) correspond to the basic atoms of the unit cell, which are considered to determine the unit cell itself. Herewith, it is assumed that $\mathbf{r}_{0}=\mathbf{0}$. For $\alpha \neq 0$, the vectors $\mathbf{r}_{\alpha}$ are defined by the obvious relations $\mathbf{r}_{\alpha}=x_{\alpha \xi} \mathbf{e}_{\xi}$, where $x_{\alpha \xi}$ are the coordinates of the atom for $\alpha \neq 0$.

Here, we will analyze only the general features of the possible influence of the complex composition of the unit cell on the dynamical properties of quasiparticles that do not require a detailed consideration of the lattice structure. In this case, by using the procedure of Hamilton dynamical minimization $[4,16]$, $i \hbar \frac{\partial a_{\mathbf{n}_{\alpha}}}{\partial t}=\frac{\partial E(\{a\})}{\partial a_{\mathbf{n}_{\alpha}}^{*}}$, we get the system of equations

$i \hbar \frac{\partial a_{\mathbf{n}_{\alpha}}}{\partial t}=D_{\mathbf{n}_{\alpha}} a_{\mathbf{n}_{\alpha}}+\sum_{\mathbf{m}_{\beta}\left(\neq \mathbf{n}_{\alpha}\right)} M_{\mathbf{n}_{\alpha} \mathbf{m}_{\beta}} a_{\mathbf{m}_{\beta}}$.

In the approximation of an ideal lattice, Eq. (19) reduces to

$i \hbar \frac{\partial a_{\mathbf{n}_{\alpha}}}{\partial t}=D_{\alpha} a_{\mathbf{n}_{\alpha}}+\sum_{\mathbf{m}_{\beta}\left(\neq \mathbf{n}_{\alpha}\right)} M_{\mathbf{m}_{\beta}-\mathbf{n}_{\alpha}} a_{\mathbf{m}_{\beta}}$.

In view of representation (18), this system can be presented as

$i \hbar \frac{\partial a_{\mathbf{n}_{\alpha}}}{\partial t}=D_{\alpha} a_{\mathbf{n}_{\alpha}}+\sum_{\mathbf{m}_{\beta}\left(\neq \mathbf{n}_{\alpha}\right)} M_{\mathbf{m}-\mathbf{n}+\mathbf{r}_{\beta}-\mathbf{r}_{\alpha}} a_{\mathbf{m}_{\beta}}$.
Now, it is convenient to rewrite (20) as follows:

$$
\begin{aligned}
& i \hbar \frac{\partial a_{\mathbf{n}_{\alpha}}}{\partial t}=D_{\alpha} a_{\mathbf{n}_{\alpha}}+\sum_{\beta(\neq \alpha)} M_{\mathbf{r}_{\beta}-\mathbf{r}_{\alpha}} a_{\mathbf{n}_{\beta}}+ \\
& +\sum_{\mathbf{m}(\neq \mathbf{n})} M_{\mathbf{m}-\mathbf{n}} a_{\mathbf{m}_{\alpha}}+\sum_{\mathbf{m}(\neq \mathbf{n})} \sum_{\beta(\neq \alpha)} M_{\mathbf{m}-\mathbf{n}+\mathbf{r}_{\beta}-\mathbf{r}_{\alpha}} a_{\mathbf{m}_{\beta}} .
\end{aligned}
$$

The solution of this equation is usually sought in the form

$a_{\mathbf{n}_{\alpha}}=A_{\alpha} e^{i[(\mathbf{k} \cdot \mathbf{n})-\omega t]}$,

where, unlike the case of a simple lattice (one atom per unit cell), the coefficients $A_{\alpha}$ are complex quantities. Then Eq. (21) takes the form of an algebraic system for the coefficients $A_{\alpha}$ and $A_{\alpha}^{*}$ :

$$
\begin{aligned}
& \left(D_{\alpha}+\sum_{\mathbf{n}(\neq \mathbf{0})} M_{\mathbf{n}} e^{i(\mathbf{k} \cdot \mathbf{n})}-\hbar \omega\right) A_{\alpha}+\sum_{\beta(\neq \alpha)} M_{\mathbf{r}_{\beta}-\mathbf{r}_{\alpha}} A_{\beta}+ \\
& +\sum_{\beta(\neq \alpha)} A_{\beta} \sum_{\mathbf{n}(\neq \mathbf{0})} M_{\mathbf{n}+\mathbf{r}_{\beta}-\mathbf{r}_{\alpha}} e^{i(\mathbf{k} \cdot \mathbf{n})}=0 ; \\
& \left(D_{\alpha}+\sum_{\mathbf{n}(\neq \mathbf{0})} M_{\mathbf{n}} e^{-i(\mathbf{k} \cdot \mathbf{n})}-\hbar \omega\right) A_{\alpha}^{*}+\sum_{\beta(\neq \alpha)} M_{\mathbf{r}_{\beta}-\mathbf{r}_{\alpha}} A_{\beta}^{*}+ \\
& +\sum_{\beta(\neq \alpha)} A_{\beta}^{*} \sum_{\mathbf{n}(\neq \mathbf{0})} M_{\mathbf{n}+\mathbf{r}_{\beta}-\mathbf{r}_{\alpha}} e^{-i(\mathbf{k} \cdot \mathbf{n})}=0 .
\end{aligned}
$$

The term $\sum_{\mathbf{n}(\neq \mathbf{0})} M_{\mathbf{n}} e^{ \pm i(\mathbf{k} \cdot \mathbf{n})}$ describes the processes of translation of an excitation between atoms of different elementary cells, which correspond to any of indices $\alpha$. If the approximation of nearest neighbors is used, then this sum is usually equal to zero.

The term $M_{\mathbf{r}_{\beta}-\mathbf{r}_{\alpha}}$ describes the processes of translation of an excitation between nonequivalent atoms of the same elementary cell. These terms are generally determine the degree of complexity of a specific band structure of the crystal.

Finally, the terms $\sum_{\mathbf{n}(\neq \mathbf{0})} M_{\mathbf{n}+\mathbf{r}_{\beta}-\mathbf{r}_{\alpha}} e^{ \pm i(\mathbf{k} \cdot \mathbf{n})}$ describe the translation process of an excitation between atoms of different elementary cells nonequivalent to one another. These terms determine mainly the properties of each of the energy bands.

Non-trivial solutions of system (23) are determined by the conditions of its compatibility, i.e. by the equality of its determinant to zero. However, this condition can be realized only under the specification of the crystal lattice.

ISSN 2071-0194. Ukr. J. Phys. 2016. Vol. 61, No. 6 
Below, we will consider a simple lattice (one atom per unit cell) in order to be able to focus on the general dynamical properties of quasiparticles. A complex lattice structure will be considered only in Subsection 3.3.2 to illustrate the use of system (23) and some features of the dynamics of quasiparticles in the case of graphene. In particular, a complicated unit cell with two atoms, instead of two nested simple sublattices, as is usually done. will be considered.

\section{3. "Mechanical branch" of a dynamical description for crystals with simple unit cell (in relation to a mechanical momentum $\mathbf{P}_{m}$ )}

To obtain the property $E(\mathbf{k}) \equiv L_{m}(\mathbf{V})$, which is given in Subsection 2.1, it is necessary to eliminate the wave vector $\mathbf{k}$ in (16) by definition (10). We obtain

$\varepsilon(\mathbf{k}) \equiv L_{m}(\boldsymbol{\beta})=-m C^{2} \sum_{i} \sqrt{1-\beta_{i}^{2}}$.

Using definitions (10) and (11) with regard for the relation $P_{i(m)}(\mathbf{k})=m_{i j}(\mathbf{k}) V_{j}(\mathbf{k})$, which is given in Subsection 2.1, and taking into account that the identity $\cos \left(b k_{i}\right) \equiv \sqrt{1-\beta_{i}^{2}}$ holds under three abovementioned approximations, we obtain

$P_{i(m)}=\frac{m V_{i}}{\cos \left(b k_{i}\right)} \equiv m C \frac{\beta_{i}}{\sqrt{1-\beta_{i}^{2}}}$.

The appearance of the factor $\sqrt{1-\beta_{i}^{2}}$ in dynamical relations under the excitation of condensed systems was also mentioned in other investigations (in particular, in $[3,19])$.

The definitions of Lagrangian (24) and momentum (25) allow us to consider the mechanical description of the dynamics of quasiparticles to be completed. It is remains only to write the mechanical Hamiltonian in the standard way: $H_{m}\left(\mathbf{P}_{m}\right)=C \sum_{i} \beta_{i} P_{i(m)}-$ $-L_{m}(\boldsymbol{\beta})$. As a result, we obtain the following definition: $H_{m}\left(\mathbf{P}_{m}\right)=m C^{2} \sum_{i} \sqrt{1+\left(p_{i(m)}\right)^{2}}$, where is marked: $p_{i(m)} \equiv P_{i(m)} / m C=\beta_{i} / \sqrt{1-\beta_{i}^{2}}$. Here, $p_{i(m)}$ are the components of the dimensionless mechanical momentum.

\section{4. "Wave branch"of a dynamical description in crystals with simple unit cell (in relation to a wave momentum $\mathrm{P}$ )}

For the final construction of this description, we have the definition of Hamiltonian (17), whose dimen- sionless form reads $h(\mathbf{p})=-\sum_{i} \cos \left(p_{i}\right)$, and relation (12) between a speed and a wave momentum: $\beta_{i}=\sin \left(p_{i}\right)$. The dimensionless wave momenta are defined by the equation

$p_{i}=b k_{i}$.

It is not difficult to show, by using the definition $P_{i}=\hbar k_{i}$ and definition (15) written in the form $m C=\hbar / b$ that $b k_{i}=\frac{b}{\hbar} P_{i}=\frac{P_{i}}{m C}=p_{i}$. To complete this description, it is enough to define the proper Lagrangian. Again, we will use the standard definition

$l(\boldsymbol{\beta})=\sum_{i} \beta_{i} p_{i}-h(\mathbf{p})$.

As a result, we have

$l(\boldsymbol{\beta})=\sum_{i}\left(\beta_{i} \arcsin \left(\beta_{i}\right)+\sqrt{1-\beta_{i}^{2}}\right)$.

It is obvious that $l(\boldsymbol{\beta}) \equiv L(\boldsymbol{\beta}) / m C^{2}$. Lagrangian $(28)$ is a new result. It belongs to the "wave branch" of a dynamical description of the object, unlike the mechanical Lagrangian (24), whose dimensionless form is as follows: $l_{m}(\boldsymbol{\beta})=-\sum_{i} \sqrt{1-\beta_{i}^{2}}$. Lagrangian (28) at the implementation of the operations $\partial l(\boldsymbol{\beta}) / \partial \beta_{i}$ generates, indeed, the components of the wave momentum: $p_{i}=\arcsin \left(\beta_{i}\right)$. The last equality is inverse relative to (12) (if we use $b k_{i}=p_{i}$ ). The necessity to carry out the inverse transformation means the limitation on the components of the momentum $p_{i}$ by such inequalities: $\left|p_{i}\right| \leq \pi / 2$.

Thus, for the consideration of a quasiparticle, as a classic object (material point), we have two descriptions equal in rights. One of them (mechanical) can be called the relativistic approximation, as it practically have the same form and was got in three approximations listed above (plane wave in a phase, nearest neighbors, and cubic lattice). Another description (wave) has the Hamiltonian

$h(\mathbf{p})=-\sum_{i} \cos \left(p_{i}\right)$

and Lagrangian (28). In the general case, it is impossible to do the choice between these descriptions without additional researches. Except for, perhaps, the relativistic approximation, which is considered here and in which the mechanical Lagrangian $l_{m}(\boldsymbol{\beta})=-\sum_{i} \sqrt{1-\beta_{i}^{2}}$ and the mechanical Hamiltonian $h_{m}\left(\mathbf{p}_{m}\right)=\sum_{i} \sqrt{1+\left(p_{i(m)}\right)^{2}}$ have the familiar "relativistic" form. 


\section{Results and Discussion}

\subsection{Generalized de Broglie ratio in crystals with simple unit cell}

Here, we will stop on the general relation between the momenta $\mathbf{P}$ and $\mathbf{P}_{m}$. Using definition (12) in equality (25) and replacing components $k_{i}$ by the components $P_{i}$ in accordance with the definition: $k_{i}=P_{i} / \hbar$, we get $P_{i(m)}=m C \operatorname{tg}\left(P_{i} / m C\right)$. This can be called the generalized de Broglie relation. While deducing the dependence $P_{i(m)}$ on $P_{i}$, the definition of mass (15) written down in the form $m C=\hbar / b$ was used again. This definition, as mentioned above, yields the definition of dimensionless wave momentum: $p_{i} \equiv$ $\equiv P_{i} / m C \equiv b k_{i}$. Now, it is obvious that the correspondence between the mechanical momentum and its operator representation is more complicated than for the wave momentum: $\widehat{P}_{j(m)}=-i m C \operatorname{th}\left((\hbar / m C) \nabla_{\mathbf{n}}^{j}\right)$, where $\nabla_{\mathbf{n}}^{j} \equiv \partial / \partial x_{j}$. On the other hand, with an account of $(15): \widehat{P}_{j(m)}=-i(\hbar / b)$ th $\left(b \nabla_{\mathbf{n}}^{j}\right)$. The relation between momenta can be represented in the dimensionless form:

$p_{i(m)}=\operatorname{tg}\left(p_{i}\right)$.

Obviously, equality (30) becomes the de Broglie ordinary relation $\mathbf{p}_{m}=\mathbf{p}$ only in the case of very small momenta and, accordingly, very small speeds. The relation $\mathbf{p}_{m}=\mathbf{p}$ looks here as the zero approximation.

Relation (30) is a new result, which is, indeed, a generalized de Broglie correlation in the approximations used here (plane wave in a phase, the nearest neighbors, and the cubic lattice). In more complex cases (concerning the structure of the lattice), this ratio will be another. But the approximation $\mathbf{p}_{m}=\mathbf{p}$ must always be applied, if all the dynamical characteristics are small.

\subsection{Relation of classic descriptions to the quantum description in crystals with simple unit cell}

The quantum description in three accepted approximations is related to the wave function (7). In these approximations, it takes such a form:

$a_{\mathbf{n}}(t)=\frac{1}{\sqrt{N}} \exp \left[i\left(p_{j} x_{j}-h(\mathbf{p}) \tau\right)\right]$.

The variables $x_{j}$ are components of the vector of lattice $\mathbf{n}=\{b x, b y, b z\}$ in the approximation of cubic lattice. The Hamiltonian $h(\mathbf{p})$ was defined in (29). The dimensionless time $\tau$ has such definition: $\tau \equiv\left(m C^{2} / \hbar\right) t=(|M| / \hbar) t$. Since function (31) is formulated in terms of the wave momentum, it gives advantage at once to a "wave branch" description of the classical type.

The quantum description is always formulated in an own coordinate system. To formulate solution (31) in this system, we must find, at first, the point of conditional localization of the excitation. Since a quasiparticle has a speed $\boldsymbol{\beta}=\left\{\beta_{j}\right\}$, its conditional location at the time moment $\tau$ can be set by the vector $\mathbf{x}_{0}$ with components: $x_{0}^{j} \equiv \beta_{j} \tau$. With regard for the definition of the relative variables: $x_{j}-x_{0}^{j} \equiv \xi_{j}$ and the common definition of wave Lagrangian $l(\boldsymbol{\beta})=\sum_{i} \beta_{i} p_{i}-h(\mathbf{p})$, we obtain $a_{\mathbf{n}}(t)=\frac{1}{\sqrt{N}} \exp \left[i\left(p_{j} \xi_{j}+l(\boldsymbol{\beta}) \tau\right)\right]$. The phase part of the solution is actually disintegrated into two multipliers. The first one, $\exp [i(\mathbf{p} \cdot \xi)]$, is the stationary quantum wave function of a free particle in the own coordinate system related to the point $\mathbf{x}_{0}$. This point moves relative to the crystal lattice with speed $\boldsymbol{\beta}$. The second multiplier $\exp (i l(\boldsymbol{\beta}) \tau)$ depends on the classical action $S(\tau)=\int_{0}^{\tau} l\left(\beta^{\prime}\right) d \tau^{\prime}$. In the case of free motion, this action reduces to the form $S(\tau)=l(\boldsymbol{\beta}) \tau$. It determines the dynamics of the point $\mathbf{x}_{0}$. Such description is always presented in the phase of the wave function as a separate term and always has the meaning of the classical action of the object.

\subsection{The dynamical Dirac model for graphenes as an approximation in the generalized dynamical description}

\subsubsection{Simple unit cell}

It is known $[21,22]$ that, in graphenes, the dispersion properties of electronic excitations $E(\mathbf{p})$ have two features. One of these features is caused by the lattice structure of a crystal. This feature leads to the appearance of up to four coupled dispersion relations. The second feature is caused by a series expansion of the dispersion relations near a point, where the components of the dimensionless wave momentum takes values $\left|p_{i}\right|=\pi / 2$. As a result, this leads to a formal description, which resembles the Dirac theory of electrons. Here, we consider the crystals with a simple lattice. That is why we will stop only on the second feature - on a Taylor series expansion. For a greater physical clarity, we will consider the excitation energy 
in the form (17), but in a partly dimensionless form: $H(\mathbf{p})=-m C^{2} \sum_{i} \cos \left(p_{i}\right),(i=\{x, y, z\})$.

At the point $\left|p_{i}\right|=\pi / 2$, we can get a series expansion linear in $\mathbf{p}$ :

$H(\mathbf{p})=m C^{2}\left(\sum_{i} p_{i}\right)-3 \pi m C^{2} / 2$.

It is easy to verify that, for two-dimensional crystals of the type of graphenes, this energy is reduced to the form $H(\mathbf{p})=m C^{2}\left(\sum_{i} p_{i}\right)-\pi m C^{2}$, where $i=\{x, y\}$. But it can be shown that, at any point $\mathbf{p}=\mathbf{p}_{0}$, except for the point $\mathbf{p}=\mathbf{0}$, one can get a linear representation

$H(\mathbf{p})=m C^{2}\left(\boldsymbol{\beta}_{0} \cdot \mathbf{p}\right)-L\left(\boldsymbol{\beta}_{0}\right)$.

If $\mathbf{p}_{0}$ is a fixed vector with components $p_{0}^{i}$, then $\boldsymbol{\beta}_{0} \equiv \mathbf{e}_{i} \sin \left(p_{0}^{i}\right) ; \mathbf{e}_{i}$ are the unit vectors of the coordinate axes of the crystal; $L\left(\boldsymbol{\beta}_{0}\right) \equiv m C^{2} l\left(\boldsymbol{\beta}_{0}\right)$, and the definition of $l\left(\boldsymbol{\beta}_{0}\right)$ is given in (28).

\subsubsection{General case}

In this paragraph, we will stop in details on the applicability of Eq. (23) to a real graphene. The main purpose of the return to the well-investigated question of the crystal and energy electronic structure of graphene lies in two circumstances. The first aim consists in the consideration based on a complicated elementary cell, rather than on two simple sublattices (consideration based on a complicated elementary cell is rarely used). The second aim is to demonstrate a more important fact of the possibility to use a linear approximation, relative to the wave momentum, not only at the points of contact of the energy bands, but practically at any point of the k-space (except for the point $\mathbf{k}=\mathbf{0}$, if the extremum at it is implemented). The figure shows a fragment of the graphene lattice and its unit cell with two atoms.

The two-dimensional lattice vector is defined by the equality $\mathbf{n}=n_{1} \mathbf{b}_{1}+n_{2} \mathbf{b}_{2}$. The elementary cell, which is shown in detail in the Figure, corresponds to the values $n_{1}=n_{2}=0$. From the geometry of the unit cell, we can see that $\mathbf{b}_{1}=\mathbf{e}_{x} b, \mathbf{b}_{2}=\mathbf{e}_{x} \frac{1}{2} b+\mathbf{e}_{y} \frac{3}{2} a$, where $b=a \sqrt{3}$. Then we get for the vector $\mathbf{n}: \mathbf{n}=$ $=\mathbf{e}_{x}\left(n_{1}+\frac{1}{2} n_{2}\right) b+\mathbf{e}_{y} \frac{3}{2} n_{2} a$. Given $\mathbf{k}=\mathbf{e}_{x} k_{x}+\mathbf{e}_{y} k_{y}$, the scalar product $(\mathbf{k} \cdot \mathbf{n})$ in the definition of the factors $e^{ \pm i(\mathbf{k} \cdot \mathbf{n})}$ of system (23) has the form $(\mathbf{k} \cdot \mathbf{n})=$ $=n_{1} p_{1}+n_{2} p_{2}$. Here, the dimensionless momenta $p_{1}$

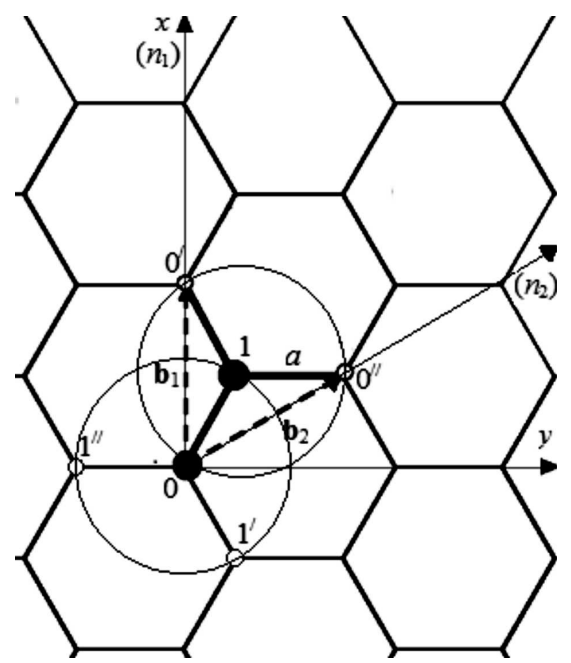

Crystal lattice of graphene and its elementary cell. Basic distance between atoms is denoted by $a$. The vectors $\mathbf{b}_{1}$ and $\mathbf{b}_{2}$ determine the crystallographic directions. The unit cell contains two atoms, which are marked by the numerals " 0 " (basic atom) and " 1 " (an extra atom). Numerals " 0 " " and " 0 " " denote the atoms of neighboring unit cells equivalent to atom " 0 ". Numerals " 1 " and " 1 " " denote the atoms of neighboring unit cells equivalent to atom " 1 ". Two circles centered at atoms " 0 " and " 1 " show the first two coordinate "spheres", which limit the consideration by the approximation of nearest neighbors

and $p_{2}$ are introduced in accordance with definitions (26) and have the form $p_{1}=\left(\mathbf{k} \cdot \mathbf{b}_{1}\right)=k_{x} b \equiv p_{x}$; $p_{2}=\left(\mathbf{k} \cdot \mathbf{b}_{2}\right)=\frac{1}{2} p_{x}+\frac{3}{2} p_{y}$, where $p_{y} \equiv k_{y} a$. Below, all will be considered relative to the momenta $p_{1}$ and $p_{2}$. The momenta $p_{x}$ and $p_{y}$ will be used for illustrative purposes.

Considering the parameters of the lattice, which is shown in the Figure, one can obtain in the nearest neighbor approximation (within the framework of two first coordination "spheres" shown in the Figure):

$$
\begin{aligned}
& \left(D_{0}-\hbar \omega\right) A_{0}+M_{a}\left(1+e^{-i p_{1}}+e^{-i p_{2}}\right) A_{1}=0 \\
& \left(D_{1}-\hbar \omega\right) A_{1}+M_{a}\left(1+e^{i p_{1}}+e^{i p_{2}}\right) A_{0}=0 \\
& \left(D_{0}-\hbar \omega\right) A_{0}^{*}+M_{a}\left(1+e^{i p_{1}}+e^{i p_{2}}\right) A_{1}^{*}=0 \\
& \left(D_{1}-\hbar \omega\right) A_{1}^{*}+M_{a}\left(1+e^{-i p_{1}}+e^{-i p_{2}}\right) A_{0}^{*}=0 .
\end{aligned}
$$

In the representation $e^{ \pm i p_{1,2}}=\cos \left(p_{1,2}\right) \pm i \sin \left(p_{1,2}\right)$, system (32) can be written in the matrix form [11, 12 , $21-23]$, as is often done:

$$
\begin{aligned}
& M_{a}\left\{1+\cos \left(p_{1}\right)+\cos \left(p_{2}\right)\right\} \widehat{\Sigma}_{1} \mathbf{A}+ \\
& +M_{a}\left\{\sin \left(p_{1}\right)+\sin \left(p_{2}\right)\right\} \widehat{\Sigma}_{2} \mathbf{A}+\widehat{D} \mathbf{A}=\hbar \omega \widehat{I} \mathbf{A}
\end{aligned}
$$


where

$$
\begin{aligned}
\mathbf{A} & =\left(\begin{array}{l}
A_{0} \\
A_{1} \\
A_{0}^{*} \\
A_{1}^{*}
\end{array}\right) ; \quad \widehat{\Sigma}_{1}=\left(\begin{array}{llll}
0 & 1 & 0 & 0 \\
1 & 0 & 0 & 0 \\
0 & 0 & 0 & 1 \\
0 & 0 & 1 & 0
\end{array}\right) \equiv\left(\begin{array}{ll}
\widehat{\sigma}_{x} & 0 \\
\widehat{0} & \widehat{\sigma}_{x}
\end{array}\right) ; \\
\widehat{\Sigma}_{2} & =\left(\begin{array}{llll}
0 & -i & 0 & 0 \\
i & 0 & 0 & 0 \\
0 & 0 & 0 & i \\
0 & 0 & -i & 0
\end{array}\right) \equiv\left(\begin{array}{lll}
\widehat{\sigma}_{y} & 0 \\
\widehat{0} & -\widehat{\sigma}_{y}
\end{array}\right) ; \\
\widehat{D} & =\left(\begin{array}{llll}
D_{0} & 0 & 0 & 0 \\
0 & D_{1} & 0 & 0 \\
0 & 0 & D_{0} & 0 \\
0 & 0 & 0 & D_{1}
\end{array}\right) ; \quad \widehat{I}=\left(\begin{array}{llll}
1 & 0 & 0 & 0 \\
0 & 1 & 0 & 0 \\
0 & 0 & 1 & 0 \\
0 & 0 & 0 & 1
\end{array}\right) .
\end{aligned}
$$

We note that the matrices $\widehat{\Sigma}_{1}$ and $\widehat{\Sigma}_{2}$ can be represented via the Pauli matrices $\widehat{\sigma}_{x}$ and $\widehat{\sigma}_{y}$.

Like Subsection 3.3.1, a linear representation relatively to the momentum $\mathbf{p}$ can be obtained in the neighborhood of any point $\mathbf{p}_{0} \equiv\left\{p_{1}^{0}, p_{2}^{0}\right\}$, which differs from the point $\mathbf{p}_{0}=\mathbf{0}$ :

$$
\begin{aligned}
& p_{1}\left\{\beta_{1}^{0} \widehat{\Sigma}_{1} \mathbf{A}-\frac{1}{\mu_{1}^{0}} \widehat{\Sigma}_{2} \mathbf{A}\right\}+p_{2}\left\{\beta_{2}^{0} \widehat{\Sigma}_{1} \mathbf{A}-\frac{1}{\mu_{2}^{0}} \widehat{\Sigma}_{2} \mathbf{A}\right\}- \\
& -\left\{l\left(\boldsymbol{\beta}_{0}\right)+1\right\} \widehat{\Sigma}_{1} \mathbf{A}- \\
& -\left\{\beta_{1}^{0}-\frac{1}{\mu_{1}^{0}} p_{1}^{0}+\beta_{2}^{0}-\frac{1}{\mu_{2}^{0}} p_{2}^{0}\right\} \widehat{\Sigma}_{2} \mathbf{A}+\widehat{G} \mathbf{A}=\Omega \widehat{I} \mathbf{A} .
\end{aligned}
$$

To provide a more compact recording and to demonstrate the connection of the obtained linear representation with the dynamical characteristics of quasiparticles, the following definitions were taken into account in Eq. (33): definition (12) for the dimensionless speed $\beta_{i}^{0}=\sin \left(p_{i}^{0}\right)$; definition (28) for the dimensionless wave Hamiltonian $h\left(\mathbf{p}_{0}\right)=$ $=-\sum_{i} \cos \left(p_{i}^{0}\right)$; definition (27) for the dimensionless wave Lagrangian $l\left(\boldsymbol{\beta}_{0}\right)=\sum_{i} \beta_{i}^{0} p_{i}^{0}-h\left(\mathbf{p}_{0}\right)$; and the definition of the tensor components of the dimensionless dynamical mass [17]: $\cos \left(p_{i}^{0}\right)=1 / \mu_{i}^{0}$. We used also the relation $M_{a}=-\left|M_{a}\right|$ typical of the crystals [16], [17] and introduced the notations $\widehat{G} \equiv \frac{1}{\left|M_{a}\right|} \widehat{D}$, $\Omega \equiv \frac{\hbar \omega}{\left|M_{a}\right|}$.

Equation (33) includes the dynamical masses $\mu_{i}^{0}$, which disappear (due to the conditions $\left(1 / \mu_{i}^{0}\right)=0$ ) only at the points of contact of the energy bands, where $p_{x}^{0}=\frac{\pi}{2}, p_{y}^{0}=\frac{\pi}{6}$ and, respectively, $\mathbf{p}_{0}=\left\{\frac{\pi}{2}, \frac{\pi}{2}\right\}$, $\boldsymbol{\beta}_{0}=\{1,1\}, l\left(\mathbf{p}_{0}\right)=\pi$. At these points, Eq. (33) is simplified up to the form

$\left(p_{1}+p_{2}\right) \widehat{\Sigma}_{1} \mathbf{A}-(\pi+1) \widehat{\Sigma}_{1} \mathbf{A}-2 \widehat{\Sigma}_{2} \mathbf{A}+\widehat{G} \mathbf{A}=\Omega \widehat{I} \mathbf{A}$.
Relative to the momenta $p_{x}$ and $p_{y}$, this equation looks like

$$
\begin{aligned}
& \left(p_{x}+p_{y}\right) \widehat{\Sigma}_{1} \mathbf{A}-(2 / 3)(\pi+1) \widehat{\Sigma}_{1} \mathbf{A}-(4 / 3) \widehat{\Sigma}_{2} \mathbf{A}+ \\
& +(2 / 3) \widehat{G} \mathbf{A}=(2 / 3) \Omega \widehat{I} \mathbf{A} .
\end{aligned}
$$

To determine the energy eigenvalues $\hbar \omega$, it is necessary to return to system (32). The compatibility condition for this system gives $\left[\left(D_{0}-\hbar \omega\right)\left(D_{1}-\hbar \omega\right)-\right.$ $\left.-M_{a}^{2}\left(1+e^{-i p_{1}}+e^{-i p_{2}}\right)\left(1+e^{i p_{1}}+e^{i p_{2}}\right)\right]^{2}=0$. From whence, we obtain

$$
\begin{aligned}
& \left(D_{0}-\hbar \omega\right)\left(D_{1}-\hbar \omega\right)= \\
& =M_{a}^{2}\left\{1+2\left[1+\cos \left(p_{1}\right)+\cos \left(p_{2}\right)+\cos \left(p_{2}-p_{1}\right)\right]\right\} .
\end{aligned}
$$

If $D_{0}=D_{1}=D$ (this is realized only under the ideal conditions of infinite crystal), then $\hbar \omega_{ \pm}=D \pm$ $\pm M_{a} \sqrt{1+2\left(1+\cos \left(p_{1}\right)+\cos \left(p_{2}\right)+\cos \left(p_{2}-p_{1}\right)\right)}$. In the $\left\{p_{x}, p_{y}\right\}$-representation for this energy, we obtain $\hbar \omega_{ \pm}=D \pm M_{a}\left\{1+2\left(1+\cos \left(p_{x}\right)+\cos \left(\frac{3}{2} p_{y}+\right.\right.\right.$ $\left.\left.\left.+\frac{1}{2} p_{x}\right)+\cos \left(\frac{3}{2} p_{y}-\frac{1}{2} p_{x}\right)\right)\right\}^{1 / 2}$. Using the cosine transformation for the sum and the difference of arguments, as well as for the cosine of double argument, we can obtain the standard expression [23] (to within the notations of parameters and axes): $\Delta E_{ \pm}=$ $= \pm M_{a} \sqrt{1+4 \cos ^{2}\left(p_{x} / 2\right)+4 \cos \left(p_{x} / 2\right) \cos \left(3 p_{y} / 2\right)}$, where $\Delta E_{ \pm} \equiv \hbar \omega_{ \pm}-D$.

\section{Conclusions}

The basic principles of construction of the general dynamical properties for quasiparticles under the excitation of materials with the structure of solids (crystals) have been analyzed. Most of the results obtained in this research are related to the crystals with a simple cubic lattice. But it is shown that these results can be considered as a zero (base) approximation for the analysis of the general dynamic properties of excited nano-objects and crystals with more complex structures of a crystal lattice. In particular, on the example of the application of the results obtained for the crystals with complex unit cell to graphene, it is shown that the Hamiltonian (Dirac representation) linear in the momentum may be valid for any crystal and for any point of the $\mathbf{k}$-space, except for the points, where the dispersive ratio have extrema.

The excitation is realized as the dispersive dependence $E(\mathbf{k})$ or $\omega(\mathbf{k})$. In order to focus attention on

ISSN 2071-0194. Ukr. J. Phys. 2016. Vol. 61, No. 6 
the general dynamical properties, the results of analysis in most cases are illustrated on the example of the simplest electronic excitations of crystals. These excitations, in particular, include the injection of an electron into the conduction band of a semiconductor, or they can be of the type of a Frenkel exciton in materials with the structure of molecular crystals.

It is shown that such excitations can be simultaneously described by three methods.

The first one is the quantum (basic) method, which gives the description of the examined excitations in terms of wave functions and eigenvalues of energy.

The second method is classical. It arises from the quantum method and is formulated in terms of a wave momentum. This second description exists in the phase of the wave function as an element, whose sense is fully identical to the classic action for the trajectory of a point of conditional localization of the excitation. The description of a quasiparticle in terms of the second method can be called the wave classicaltype description.

The third method originated from the second one is also the classical-type description, but in terms of another momentum, the mechanical one. In the approximations used here (plane wave in a phase, nearest neighbors, and cubic lattice), the third method of description practically coincides with the known dynamical description of a free relativistic particle.

A question about the "relation" of both classic descriptions to the first quantum one is considered. The generalized de Broglie correlation is found. It is shown that the Dirac dynamical model in graphene is an approximation of the generalized dynamical description of quasiparticles in crystals.

The further applications of the obtained results are as follows: investigation of the dynamics of quasiparticles in an external potential field; use of the generalized de Broglie relations for the more correct conversion of the momentum of an electron at its injection into a crystal; investigations in plasma, where the dispersive dependences of the kind of $\omega(\mathbf{k})$ are used; experimental verification of the existence of an anisotropy of the dynamical mass, which supplements the anisotropy of a crystal.

1. M.F. Smith and M.B. Walker, Phys. Rev. B 67, 214509 (2003).

2. Yu.E. Lozovik and A.G. Semenov, Theor. Math. Phys. 154, 319 (2008).

ISSN 2071-0194. Ukr. J. Phys. 2016. Vol. 61, No. 6
3. A.S. Davydov, Solitons in Molecular Systems (Kluwer, Dordrecht, 1991).

4. Yu.E. Natanzon, L.S. Brizhik, and A.A. Eremko, Ukr. J. Phys. 51, 413 (2006).

5. L.S. Brizhik, Ukr. J. Phys. 48, 611 (2003).

6. A.D. Suprun and L.V. Shmeleva, Nanoscale Res. Lett. 9, 200 (2014).

7. H. Ebrahimnejad, G.A. Sawatzky, and M. Berciu, Nature Phys. 10, 951 (2014).

8. Yu.B. Gaididei and V.M. Loktev, Ukr. J. Phys. 50, 400 (2005).

9. A. Liebsch and A. Lichtenstein, Phys. Rev. Lett. 84, 1591 (2000).

10. Yu. Rapoport, V. Grimalsky, I. Iorsh et al., JETP Letters 98, 503 (2013).

11. S.D. Sarma, S. Adam, E.H. Hwang et al., Rev. Mod. Phys. 83, 407 (2011).

12. V.P. Gusynin, S.G. Sharapov, and J.P. Carbotte, Int. J. Mod. Phys. B 21, 4611 (2007).

13. O.P. Verkhoglyadova, B.T. Tsurutani, and G.S. Lakhina, J. of Geophys. Res.: Space Phys. 118, 7695 (2013).

14. A.V. Filippov, A.G. Zagorodny, A.I. Momot et al., JETP 108, 497 (2009).

15. I.M. Mryglod and V.M. Kuporov, Ukr. J. Phys. 55, 117 (2010).

16. A.D. Suprun and L.V. Shmeleva, Functional Mater. 19, $508(2012)$

17. A.D. Suprun and L.V. Shmeleva, Functional Mater. 21, 69 (2014)

18. A.D. Suprun, Theor. Math. Phys. 57, 1141 (1983).

19. V.A. Mironov, A.I. Smirnov, and L.A. Smirnov, JETP 112, 46 (2011).

20. A.S. Davydov, Theory of Molecular Excitons (Springer, Berlin, 2013).

21. Yu.A. Sitenko and N.D. Vlasii, Nucl. Phys. B 787, 241 (2007)

22. A. Attaccalite, L. Wirtz et al., Phys. Rev. B 78, 205425 (2008).

23. P.R. Wallace, Phys. Rev. 71, 622 (1947).

Received 23.09.15

\section{А.Д. Супрун, Л.В.Шмельова}

ДЕЯКІ АСПЕКТИ УЗАГАЛЬНЕНОЇ

ДИНАМІКИ КВАЗІЧАСТИНОК В КРИСТАЛАХ

3 ЕЛЕМЕНТАРНОЮ КОМІРКОЮ

ДОВІЛЬНОЇ СКЛАДНОСТІ

Р е $з$ ю м е

Проаналізовано умови, за яких загальний опис динамічних властивостей квазічастинок майже збігається з аналогічним описом для реальних релятивістських частинок. Такий аналіз на сьогодні актуальний у зв'язку із зростан- 
ням інтересу до електронних властивостей графена та інших наноструктур вуглецевого походження (фулерени, нанотрубки і таке інше). Розвиток традиційних застосувань квазічастинок (надплинність, перенос заряду або енергії) також вимагає узагальненого аналізу динамічних властивостей квазічастинок. Розглянуто проблему співвідношення квантового і класичного способів опису для збуджених станів кристалів. Для того, щоб зосередити увагу на обговорюваній проблемі, отримані результати були продемонстровані на прикладах електронних збуджень кристалів у найпростішому випадку, коли іншими ефектами нехтують (фононами, дефектами, високою щільністю збуджень, яка вимагає врахування взаємодії між ними, реакцією ґратки на збудження і таке інше). Було показано, що такі збудження описуються трьома способами одночасно. Перший - це квантовий спосіб, який дає опис розглянутих збуджень в термінах хвильових функцій і власних значень енергії. Другий спосіб - класичний. Він виникає з квантового способу і формулюється по відношенню до хвильового імпульсу. Третій спосіб, який є похідним від другого, також є описом класичного типу, але по відношенню до іншого імпульсу - механічного. Цей (третій або другий класичний) опис дозволяє інтерпретувати експериментальні дані в термінах звичайної релятивістської динаміки. 